\title{
Positive and negative compatibility effects
}

\author{
DAPHNE BAVELIER \\ University of Rochester, Rochester, New York \\ CHRISTINE DERUELLE \\ CNRS, Marseille, France \\ and \\ JASON PROKSCH \\ University of Rochester, Rochester, New York
}

\begin{abstract}
This paper reports a series of four experiments that established a negative compatibility effect (NCE) by which compatible distractors led to slower and less accurate target performance than did incompatible ones (Experiment 1). This effect is interpreted as an early perceptual effect that delays the attribution of visual attention over the target location in the compatible condition. This view predicted that the NCE should be observed only when attention has to be selectively attributed to the target location. In Experiments 2 and 3, this prediction was tested by manipulating the perceptual load in the display. High perceptual load displays are known to require selective attention (Lavie, 1995). Accordingly, reliable NCEs were observed when high-load displays were used. In contrast, reduced NCEs were found in displays that did not require selective attention. Experiment 4 established that the manifestation of the NCE was influenced by low-level visual cues, such as brightness and contrast. Overall, these experiments indicated that the NCE can be understood as an early perceptual effect, which arises from a conflict between the cues that guide the distribution of attention when the task requires selective attention.
\end{abstract}

Response to a target shape is facilitated by the presence of distractors associated with the same response (compatible distractors), as compared with distractors associated with a competing response (incompatible distractors). Participants presented with a display containing two shapes, one blue and one red, are typically slower and less accurate at identifying the blue shape as a square when the red shape is a diamond rather than a square. This effect will hereafter be termed the positive compatibility effect (PCE). Although the Stroop effect and the flanker compatibility effect are probably the most well-known examples of positive compatibility effects, compatibility effects are so pervasive that they are observed across various tasks and stimuli (see Kornblum \& Lee, 1995, for an exhaustive review of the different kinds of compatibility effects).

For this research, D.B. was supported by the McDonnell Pew Foundation for Cognitive Neuroscience, and C.D. by the Cogniscience Program, CNRS. This research was also supported in part by Grant 7-RO1DC00481 to Helen Neville, from the National Institute on Deafness and Other Communication Disorders, National Institute of Health, and by Grant DAMD17-93-V-3018 to the Georgetown Institute for Cognitive and Computational Sciences, from the Department of Defense. We thank Francois Klam, Eric Klineberg, and David Higgs for help with data collection and manuscript preparation. We are especially indebted to Mary Potter for her thorough comments on previous drafts and to Alexandre Pouget, Cees Van Leeuwen, and Lise Paquet for helpful discussions. Correspondence concerning this article should be addressed to D. Bavelier, Brain and Cognitive Sciences, Meliara Hall-0268, Rochester. NY 14627 (e-mail: daphne@bcs.rochester.edu).
Surprisingly, in a series of experiments on selective attention that manipulated a variant of the flanker compatibility effect, Briand (1994) found, under certain conditions, a negative compatibility effect (NCE). In the experiment of interest, two compound letters were presented, one in red and the other in blue (Figure 1). The participants were required to identify letters at the local level of the blue group while ignoring the red group. The participants were 29 msec slower to identify the local blue letters when the identity of the global red letter was compatible than when it was incompatible. Similarly, in a series of experiments that manipulated the Stroop effect and the Garner effect, Van Leeuwen and Bakker (1995) reported NCEs. In their experiments, participants were presented with two shapes, each with a local indentation and a global form. They were asked to decide whether the local indentation of the target shape was a triangle or a square, while the global form was manipulated to be compatible or incompatible with the target indentation. For the same visual displays, they observed either a PCE or an NCE as the display duration was varied (Van Leeuwen \& Bakker, 1995, Experiment 2, Figure 5). These results are striking, since they show that an NCE can be observed with exactly the same visual displays as those in which a PCE is expected.

The observation of an NCE is surprising within our present understanding of visual attention and visual selection. As we just reviewed, the effect of distractors has been understood as being due to the competition of distractors with the target during response selection (Stroop 


\section{COMPATIBLE}

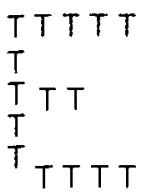

\section{INCOMPATIBLE}

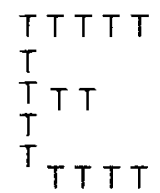

$\begin{array}{ll}L & L \\ L & L \\ L & L \\ L & L\end{array}$

Figure 1. Examples of displays manipulating the compatibility of between-group global distractors used in Briand's (1994) experiments. The participants had to identify the letter at the local level of the blue shape (i.e., local $T$ in global $E$ in this figure). $T$ and $H$ were associated with different response, whereas $L$ and $E$ were never associated with a response (neutral).

effect, flanker compatibility effect, Simon effect; see Kornblum \& Lee, 1995, for a review). Some accounts hold that the effect is due to a facilitation from compatible distractors, whereas others maintain that it is due to a hindrance from incompatible ones. However, all the accounts predict that performance should be better with compatible than with incompatible distractors. The finding that compatible distractors disrupt processing more than do incompatible ones clearly rules out accounts in terms of competition during response selection. Although the NCE may seem puzzling in regard to the previous literature on visual selection, we would like to propose that it results from the early processes that guide target selection. The goal of the experiments presented in this paper was to establish a reliable NCE, to characterize the conditions under which it is observed, and to understand its mechanism.

\section{EXPERIMENT 1}

This experiment closely replicated the study of Briand (1994). In the variation of the experimental task developed by Paquet and Merikle (1988), Briand (1994) presented two compound letter patterns (global letters made of local letters), one in red and the other in blue. The color of the pattern indicated the target. For example, in one representative session, participants had to decide whether the local letters in the blue pattern were Ts or Hs. The identities of the letters at the three levels other than the target level were systematically manipulated during this session. Distractors could appear at one of the three nontarget levels: the global level in the blue pattern (within-group global distractors), the global level in the red pattern (between-group global distractors), and the local level in the red pattern (between-group local distractors). Alternatively, all three levels could be neutral ( $\mathrm{L}$ or $\mathrm{E}$ ), providing a baseline for the experiment. Briand (1994) observed an NCE from the global letter distractors when they belonged to the red pattern (between-group condition).

The design of Experiment 1 was similar to that of Briand (1994), with the exception that the hierarchical patterns used were geometrical shapes. The standard global-to-local compatibility effect reported in the liter- ature is known to generalize to various configurations, including geometrical shapes (Fagot \& Deruelle, 1997; Navon, 1983). We presented two compound shape patterns, one in black and the other in white. Examples of the shapes presented are given in Figure 2. In this example, the participants were asked to decide whether there were local squares or local diamonds in the black pattern; circles were neutral shapes. The performance of the participants was measured as the type of the distractor varied. On the basis of the results reported by Briand (1994, Table 2, p. 568), we expected an NCE from betweengroup global distractors.

\section{Method}

Participants. Thirteen participants took part in this experiment; however, 1 participant did not meet the accuracy criterion ( $70 \%$ correct) required for this experiment and was omitted from any analysis. The 12 participants kept for analysis included 8 females and 4 males, with ages ranging from 18 to 27 years (mean age $=21$ years). All the participants were from the University of California, San Diego, community; they were right-handed and had normal or corrected-to-normal vision. The participants were paid $\$ 7$ per hour for their participation.

Stimuli and Procedure. In each trial, the participants were presented with two hierarchical objects composed of geometrical shapes. Each pattern was composed of a global shape made of local elements (Figure 2). The display was flashed on a Seiko Instrument (CM2050C) monitor $56 \mathrm{in}$. from the participant, subtending a total visual angle of $5^{\circ}$. Each hierarchical shape was displayed on a gray circle background that subtended $2.25^{\circ}$. The global level $\left(1.5^{\circ}\right)$ was always made of 12 local elements $\left(0.22^{\circ}\right)$. The patterns had different lightness; one was white, and the other was black. Half of the participants were asked to pay attention to the white group, the other half to the black group. The positions of the white and the black groups were randomly assigned, with the only constraint being that each group appeared equally often on the left and on the right of the fixation point. The experimental condition of interest consisted of a local session, during which the participants were to decide whether the local elements of the target group were squares or diamonds. The participants were also asked to perform a global session, during which they were to decide whether the global element of the target group was a square or a diamond. Order of session was counterbalanced among participants. The results of the global sessions showed the same trend as that of the local sessions. As previously reported in the global/local literature, the size of the effects were, however, attenuated. For these reasons, the global session will not be discussed further. The distractors could be located at either level (global/local) and could belong to either the target (within-group) or the nontarget (between-group) group. The identity of the distractors could be compatible, incompatible, or neutral with respect to the target. The design included all possible factorial combinations of distractor level (global/local), distractor location relative to the target group (within-group/between-group), and distractor compatibility (compatible/incompatible/neutral), for a total of seven conditions (within-group: 1 distractor level $\times 2$ distractor compatibility; between-group: 2 distractor level $\times 2$ distractor compatibility; and neutral: 1 distractor compatibility). All these factors were counterbalanced within participants.

The session comprised 280 trials (i.e., 40 trials per condition). divided into four blocks. The participants were instructed to answer as quickly as possible, while maintaining an accuracy of $95 \%$. After each block, they were given feedback about their mean accuracy and speed. Whether the participants used their right or their left hands for signaling a square or a diamond was counterbalanced 


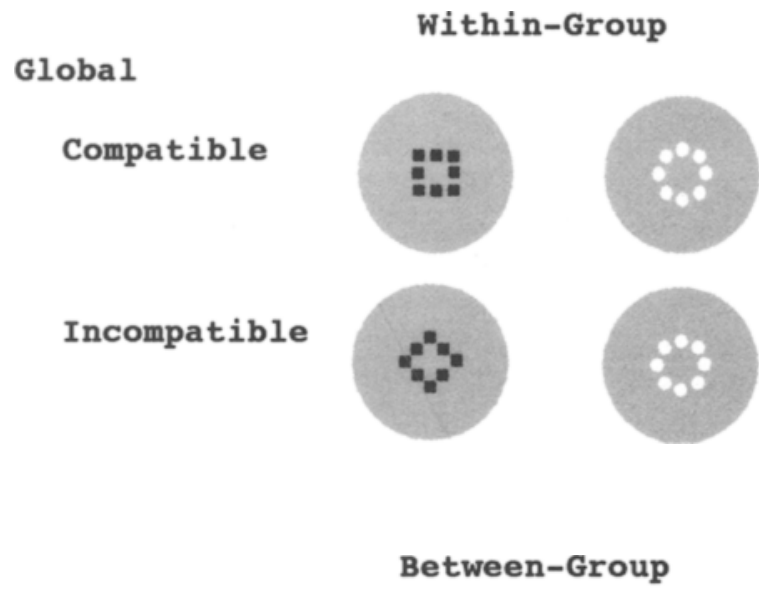

Global

Compatible

Incompatible
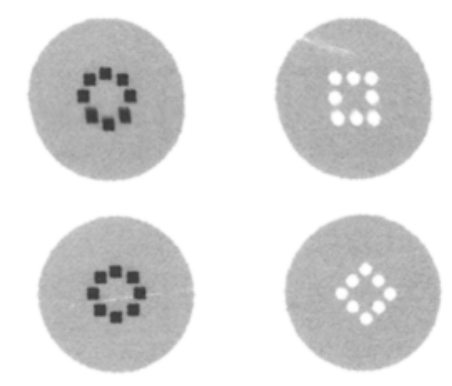

Local

Compatible

Incompatible
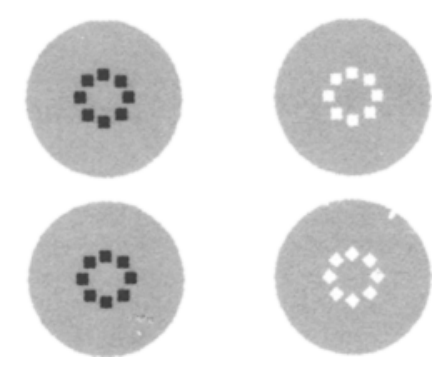

Neutral

Neutral
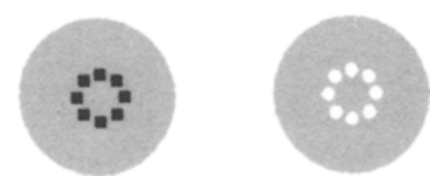

Figure 2. Examples of the stimuli used in Experiment 1 as a function of the different experimental conditions. The participants were asked to decide whether there were squares or diamonds at the local level of the black shape (only those displays in which the answer was square are shown).

across participants. Trials were self-paced; the word READY was displayed until the participant initiated the trial by pressing the space bar. A fixation dot appeared in the center of the screen for $500 \mathrm{msec}$, followed by the gray background for $500 \mathrm{msec}$ and then the stimuli for $150 \mathrm{msec}$. The onset of the stimuli activated the computer clock, which was stopped when the participant pressed one of the response keys on the response box. The intertrial interval was set to $500 \mathrm{msec}$ after the execution of the response. The participants were trained until they answered correctly for 10 consecutive trials. The session lasted about $20 \mathrm{~min}$.

\section{Results}

The mean reaction times (RTs) for correct responses were obtained for each of the 12 participants. Trials in which the RTs exceeded two standard deviations of the participant mean RT were excluded ( $4.8 \%$ of the trials). Error rates were computed and are reported in the tables. An analysis similar to those performed on mean RTs were carried out on error rates. However, error analyses did not show significant effects that differed from those revealed by RT analyses and will not be discussed further.

The mean RT was $669 \mathrm{msec}$, with a mean error rate of $10 \%$. The main results are summarized in Table 1 . The analyses focused on the compatibility effect, as measured by the difference in performance between compatible and incompatible trials. For reference, the mean RT and percent error for neutral trials are reported in Appendix A.

An analysis of variance (ANOVA), with the three different kinds of distractor (within-global/betweenglobal/between-local) and compatibility (compatible/ incompatible) as factors, revealed a main effect of distractor kind [within-global, $774 \mathrm{msec}$, vs. between-global, $791 \mathrm{msec}$, vs. between-local, $751 \mathrm{msec} ; F(2,22)=5.7$, $\left.M S_{\mathrm{e}}=1,711.2, p<.01\right]$ and no effect of compatibility $(p>.8)$. An interaction between distractor kind and compatibility indicated, however, different effects of compatibility across the three kinds of distractor $[F(2,22)=$ 23.8, $\left.M S_{\mathrm{e}}=862.6, p<.0001\right]$. This interaction led us to analyze each distractor condition separately. For withingroup global distractors, the mean RT was faster for compatible distractors $[M D=+65 \mathrm{msec} ; F(1,11)=10.5$, $\left.M S_{\mathrm{e}}=2,453.7, p<.008\right]$. This result reproduced the standard PCE observed from global distractors on local targets in the Navon configuration (Navon, 1977). In contrast, for between-group global distractors, the mean RT was slower for compatible distractors, revealing an $\mathrm{NCE}\left[M D=-49 \mathrm{msec} ; F(1,11)=21.2, M S_{\mathrm{e}}=693.8, p<\right.$ $.001]$. Finally, for between-group local distractors, the ANOVA revealed no significant effect $(M D=-11 \mathrm{msec}$, $p>.12$ ).

\section{Discussion}

As was predicted, Experiment 1 demonstrates a robust NCE from between-group global distractors. In this condition, distractors hindered target processing more when they were compatible than when they were incompatible. This result contrasts with the standard PCE from global distractors onto local targets observed in the standard Navon configuration, in which the global distractor is made up of the local targets. As in Briand (1994) and Van Leeuwen and Bakker (1995), it appears that the NCE is strongest from distractors that are global and that are not within the same perceptual group as the target.

We propose that the NCE is an early perceptual effect, which arises from a conflict between the cues that guide the distribution of attention over the display. Previous re- 
Inner-set Distractors

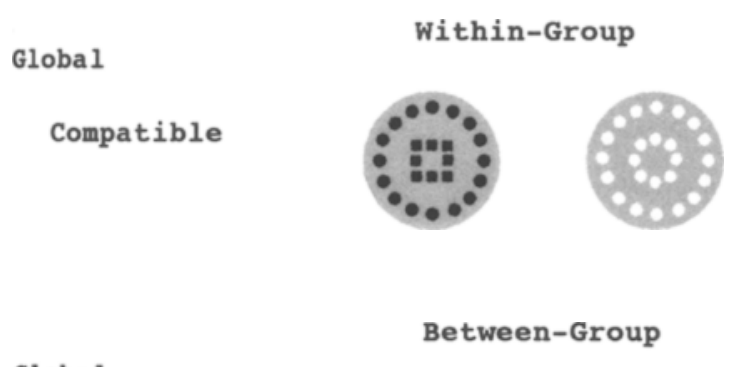

Global

Compatible

Local

Compatible
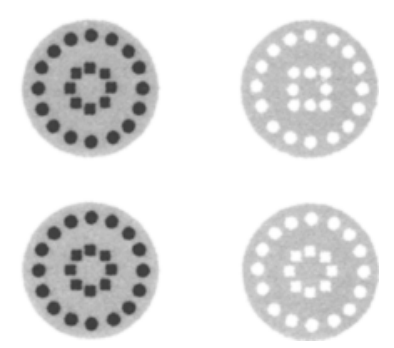

Within-Group

Global

Compatible

Local

(n)

Globa 1

Compatible
Local
Compatible

Compatible
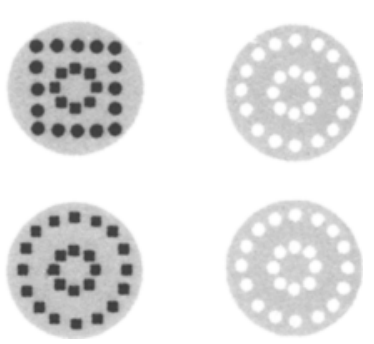

Between-Group
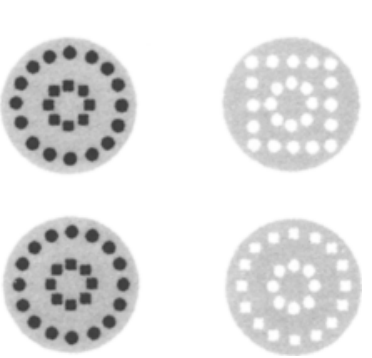

Figure 3. Examples of the stimuli used in Experiment 2 (target shape displayed in black). In this experiment, the target was always within the inner set of the target shape. Distractors could appear at any of the other seven possible locations.

search has shown that cues as varied as luminance contrast, connectedness, and other Gestalt cues that affect grouping, as well as task requirements, affect the distribution of selective attention on the display (Kramer, Coles,

\& Logan, 1995; Miller, 1982; Vecera \& Farah, 1994; Yantis, 1995). In the between-group condition of Experiment 1 , the gray backgrounds and the different lightness of the target and the distractor, along with the task requirements, suggested that the distractors and the targets should be considered separately. However, in the compatible condition, the similarity of the target and the distractors may have induced these shapes to be grouped together. We propose that this conflict among the parsing cues delays the distribution of attention over the display and is responsible for the slower RTs and the lesser accuracy seen in the compatible condition. Note that grouping by similarity is only present in the compatible condition; in the incompatible conditions, all the cues indicate that the distractors and the targets belong to unrelated groups.

This proposal holds that the NCE is due to a conflict between the factors that control the distribution of visual attention over the display. Adequate distribution of visual attention, also termed selective attention, is, however, not always necessary for target selection and processing. For example, Lavie (1995) has shown that unselective processing occurs when the target task is easy and only requires very few attentional resources. In this case, spare attentional capacities automatically spill over to process irrelevant information, and large positive effects of distractor compatibility are expected. In contrast, when the target selection is demanding and exhausts the processing capacities of the visual system, accurate target processing requires visual attention to be selectively allocated over the target location. In this case, few or no resources will be available to process distractors, leading one to predict small, if any, effects from distractors. In accordance with this proposal, Lavie has shown that low perceptual load displays that allow efficient target searches lead to greater PCEs than do high perceptual load displays, in which the target search is demanding. If the NCE is due to a conflict between factors that control the distribution of visual attention over the display, it should be robust only when the distribution of visual attention affects target performance-that is, when selective visual attention is required. In Experiments 2 and 3, this proposal was tested by manipulating the ease of target selection. We predicted that an NCE should be observed for highload displays, in which the target search is difficult, but should disappear for low-load displays, in which the target search is efficient.

\section{EXPERIMENT 2}

Experiment 2 was designed to test the hypothesis that the NCE occurs when target processing requires selective attention and is not specific to the display configurations used in Experiment 1. Experiment 2 used shapes similar to those of Experiment 1, but with the addition of an extra hierarchical level, termed the outer set. As can be seen in Figure 3, this additional level led to a substantial modification of the display configurations used in Experiment 1 . The double level of embedding is known to render target selection more difficult (Greaney \& MacRae, 
Table 1

Mean Reaction Times (RTs, in Milliseconds) and Error Rates (as Percentages) as a Function of Distractor Condition and Compatibility in Experiment 1

\begin{tabular}{lcc}
$\begin{array}{c}\text { Distractor Level } \\
\text { and Compatibility }\end{array}$ & RT & Error \\
\hline & Within-Group & \\
Global & & \\
Compatible & 742 & 14 \\
Incompatible & 807 & 15 \\
Compatibility effect & $+65 \dagger$ & +1 \\
& Between-Group & \\
Global & & 11 \\
Compatible & 816 & 4 \\
Incompatible & 767 & $-7 *$ \\
Compatibility effect & $-49 \dagger$ & 6 \\
Local & & 9 \\
Compatible & 757 & +3 \\
Incompatible & 746 & 11 \\
Compatibility effect & -11 & \\
\hline
\end{tabular}

Note-The compatibility effect was computed by subtracting the compatible score from the incompatible one. ${ }^{*} p<.05 .{ }^{\dagger} p<.01$.

1992). The proposal that the NCE is not specific to the displays used in Experiment 1 but occurs with any display in which the target search is difficult predicted reliable NCEs from between-group distractors.

\section{Method}

Participants. Thirteen participants, drawn from the same population as those in Experiment 1, took part in this experiment. None had participated in the previous experiment. One participant did not meet the $70 \%$ accuracy criterion and was omitted from any analyses. The 12 participants kept for analysis included 8 females and 4 males; their ages ranged from 18 to 30 years (mean age $=22$ years).

Stimuli and Procedure. The stimuli consisted of two patterns, each made of two hierarchical levels (Figure 3). The smaller hierarchical level was identical to that of Experiment 1; the additional level subtended about $1.9^{\circ}$ of visual angle and was always made up of 20 local elements (of $0.22^{\circ}$ each). The smaller hierarchical level will hereafter be termed the inner set, and the larger one will be termed the outer set. Apparatus, monitor settings, and procedure were otherwise identical to those in Experiment 1.

The targets were always located at the local level of the inner set (as in Experiment 1). The identities of the shapes at the seven levels other than the target level were systematically manipulated during a session. The design included all possible factorial combinations of distractor location in the hierarchical structure (outer vs. inner set), distractor level (local vs. global level), distractor location relative to the target group (within- vs. between-group location), and distractor identity (compatible, incompatible, or neutral, relative to the target), for a total of 15 different conditions. All the factors were counterbalanced within participants. The session comprised 600 trials (i.e., 40 trials per condition), divided into six blocks, and lasted about $45 \mathrm{~min}$.

\section{Results}

One of the stimulus configurations seemed abnormally difficult (within-group, outer-set, global compatible distractor, diamond targets; see Appendix B). The overall accuracy for that configuration was less than $40 \%$, whereas for all the other configurations, it was around $90 \%$. This difficulty was only found with one stimulus configuration, but not across the configurations that belonged to the same experimental condition, suggesting that the source of the problem was configuration specific. Thus, the results from this configuration were not included in the following analyses (leading to the exclusion of 20 trials $\times 12$ participants $=240$ trials). The same analysis parameters as those in Experiment 1 were otherwise used. In particular, for all 12 participants, RTs outside two standard deviations were discarded from the analysis $(2.6 \%$ of the trials). As in Experiment 1, the analyses focused on the difference between compatible and incompatible trials (Tables $2 \mathrm{~A}$ and $2 \mathrm{~B}$ ). The mean RTs and percent error for neutral trials are reported in Appendix A for reference. Error analyses will not be reported unless they indicated an effect that differed from the RT analysis.

Inner-set distractors. The mean RT was $753 \mathrm{msec}$, with a mean error rate of $11 \%$. The results are reported in Table 2A; note that the displays in this condition were similar to those of Experiment 1, except for the addition of an outer ring of circles.

An ANOVA, with the three different kinds of distractors (within-global/between-global/between-local) and compatibility (compatible, incompatible) as factors, revealed a main effect of distractor condition [within-global, $774 \mathrm{msec}$, vs. between-global, $765 \mathrm{msec}$, vs. betweenlocal, $\left.732 \mathrm{msec} ; F(2,22)=3.6, M S_{\mathrm{e}}=3,248.0, p<.045\right]$, no effect of compatibility $(p>.2)$, and an interaction between distractor kind and compatibility $[F(2,22)=$ 80.3, $\left.M S_{\mathrm{e}}=616.2, p<.0001\right]$. Thus, as in Experiment 1 , the compatibility effects varied across the three kinds of distractors. For within-group global distractors, the standard PCE was observed, in which compatible distractors led to faster RTs than did incompatible ones $[M D=$ $\left.+113 \mathrm{msec} ; F(1,11)=61.8, M S_{\mathrm{e}}=1,233.9, p<.0001\right]$. In contrast, for between-group global distractors, the mean RT was slower for compatible distractors, revealing again an NCE $\left[M D=-59 \mathrm{msec} ; F(1,11)=56.6, M S_{\mathrm{e}}=\right.$ $371.9, p<.0001]$. Between-group local distractors also led to an NCE $\left[M D=-24 \mathrm{msec} ; F(1,11)=5.5, M S_{\mathrm{e}}=\right.$ $615.5, p<.04]$. This pattern of results closely replicated that of Experiment 1 .

Outer-set distractors. The mean RT was $768 \mathrm{msec}$, with a mean error rate of $8 \%$ (Table $2 B$ ). A $2 \times 2 \times 2$ ANOVA, with the four different kinds of distractors (crossed $2 \times 2$ by group and level) and compatibility (compatible/incompatible), was carried out. The mean RT was faster for the between-group condition than for the within-group condition [ 751 vs. $800 \mathrm{msec} ; F(1,11)=$ $\left.40.2, M S_{\mathrm{e}}=1,426.3, p<.0001\right]$. This group effect indicated that target processing was less disrupted by betweengroup than by within-group distractors. The mean RT was longer for global distractors than for local ones [802 vs. $\left.751 \mathrm{msec} ; F(1,11)=45.7, M S_{\mathrm{e}}=1,380.1, p<.0001\right]$. Thus, as in previous studies, global distractors were more disruptive than local ones (Navon, 1977; Paquet \& Merikle, 1988). The mean RT was longer for compatible than for incompatible distractors, indicating an overall 
Table 2A

Mean Reaction Times (RTs, in Milliseconds) and Error Rates (as Percentages) for Inner-Set Distractors in Experiment 2

\begin{tabular}{lrc}
$\begin{array}{c}\text { Distractor Level } \\
\text { and Compatibility }\end{array}$ & RT & Error \\
\hline \multicolumn{3}{c}{ Within-Group } \\
Global & 717 & 10 \\
Compatible & 830 & 26 \\
Incompatible & $+113 \ddagger$ & $+16 \dagger$ \\
Compatibility effect & Between-Group & \\
& & \\
Global & 795 & 13 \\
Compatible & 736 & 5 \\
Incompatible & $-59 \ddagger$ & $-8 \dagger$ \\
Compatibility effect & & \\
Local & 744 & 5 \\
Compatible & 720 & 8 \\
Incompatible & $-24^{*}$ & +3 \\
Compatibility effect & & \\
\hline
\end{tabular}

${ }^{*} p<.05 .{ }^{\dagger} p<.01 . \quad{ }^{\ddagger} p<.001$.

NCE [783 vs. $770 \mathrm{msec} ; F(1,11)=7.6, M S_{\mathrm{e}}=533.3, p<$ $.0185]$. Distractor level interacted with group $[F(1,11)=$ 48.6, $\left.M S_{\mathrm{e}}=783.6, p<.0001\right]$ and compatibility $[F(1,11)=$ 5.6, $\left.M S_{\mathrm{e}}=913.3, p<.037\right]$. The interaction between group and compatibility was not significant $(p>.69)$, but the three-way interaction between distractor level, group, and compatibility was marginally significant $(p=$ .09 ). This pattern of interactions led us to analyze separately the compatibility effect for the four kinds of distractors. Between-group global distractors led to an NCE $\left[M D=-41 \mathrm{msec} ; F(1,11)=8.6, M S_{\mathrm{e}}=1,191.8, p<.013\right]$. None of the other effects reached significance ( $p$ s $>$ .33), although an error analysis revealed a PCE for within-group local distractors $[M D=+6 \% ; F(1,11)=7.9$, $\left.M S_{\mathrm{e}}=0.0026, p<.017\right]$.

\section{Discussion}

The results from the inner-set distractors of Experiment 2 confirm the main findings of Experiment 1 . A strong PCE was observed for within-group distractors. This configuration corresponded to the classical configuration used by Navon (1977), in which the global distractor is made up of the local targets. For the betweengroup distractors, robust NCEs were observed, as in Experiment 1 . The finding of a significant NCE from local inner-set distractors suggests that the effect generalizes to other types of distractors besides global ones, although the NCE certainly appears strongest from global distractors. For the outer-set location, within-group distractors failed to show a clear effect of compatibility. This may have been due to their ambiguous status, since their location and color tended to group them with the target, whereas the task required them to be ignored. For the between-group distractors, global outer-set distractors led to a robust NCE, comparable with that of inner-set distractors.
The results of Experiment 2 establish a reliable NCE for three different types of stimulus configurations (innerset global distractors, inner-set local distractors, and outer-set global distractors). This pattern of findings allows us to rule out an explanation of the NCE as being an effect specific to the global between-group distractor configuration of Experiment 1. Rather, the observation of a reliable NCE with these complex embedded hierarchical shapes is consistent with the proposal that the NCE is observed when the target task is difficult and requires selective attention. In Experiment 3, this proposal was tested directly by comparing the complex, high perceptual load displays used in Experiment 1 with displays with a lower perceptual load. The proposal that the NCE requires selective attention leads one to predict a larger NCE from global between-group distractors in the highload displays than from those in the lower load displays.

\section{EXPERIMENT 3}

Experiment 3 tested the prediction that the NCE should be observed only when the target task requires selective attention. Compatibility effects were compared, when the visual displays were made up either of complex shapes, rendering the target selection difficult, or of simple shapes, rendering the target selection easy (Figure 4). We predicted that the NCE should be larger for the high perceptual load displays, in which the shapes were complex, than for the low perceptual load displays that used simple shapes. Because Van Leeuwen and Bakker (1995) showed that the size of the NCE varies with display duration, it was important to ensure that any differences between low- and high-load displays were not due to dif-

Table 2B

Mean Reaction Times (RTs, in Milliseconds) and Error Rates (as Percentages) for Outer-Set Distractors in Experiment 2

\begin{tabular}{|c|c|c|}
\hline $\begin{array}{c}\text { Distractor Level } \\
\text { and Compatibility }\end{array}$ & RT & Error \\
\hline \multicolumn{3}{|c|}{ Within-Group } \\
\hline $\begin{array}{l}\text { Global } \\
\text { Compatible } \\
\text { Incompatible } \\
\text { Compatibility effect }\end{array}$ & $\begin{array}{r}852 \\
841 \\
-11\end{array}$ & $\begin{array}{l}10 \\
11 \\
+1\end{array}$ \\
\hline $\begin{array}{l}\text { Local } \\
\text { Compatible } \\
\text { Incompatible } \\
\text { Compatibility effect }\end{array}$ & $\begin{array}{r}759 \\
751 \\
-8\end{array}$ & $\begin{array}{c}5 \\
11 \\
+6^{*}\end{array}$ \\
\hline & n-Groul & \\
\hline $\begin{array}{l}\text { Global } \\
\text { Compatible } \\
\text { Incompatible } \\
\text { Compatibility effect }\end{array}$ & $\begin{array}{c}778 \\
737 \\
-4 I^{*}\end{array}$ & $\begin{array}{c}12 \\
6 \\
-6 \dagger\end{array}$ \\
\hline $\begin{array}{l}\text { Local } \\
\text { Compatible } \\
\text { Incompatible } \\
\text { Compatibility effect }\end{array}$ & $\begin{array}{r}742 \\
750 \\
-8 \\
\end{array}$ & $\begin{array}{r}6 \\
5 \\
-1 \\
\end{array}$ \\
\hline
\end{tabular}
${ }^{*} p<.05 .{ }^{\dagger} p<.01$. 
Low Load

Global
Compatible

Local

Compatible

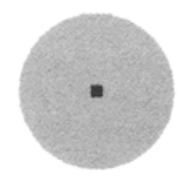

High Load
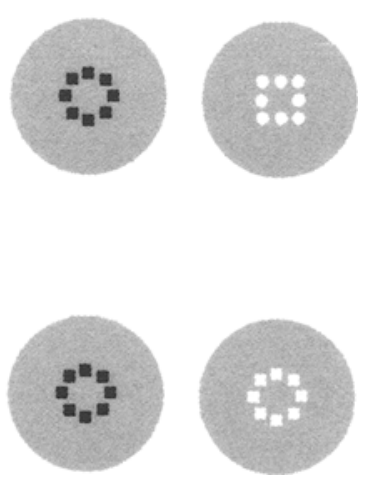

Figure 4. Example of the low-load and high-load displays used in Experiment 3. The target shape is black.

fering sensitivities to duration. To maximize chances of observing an NCE under low perceptual load, presentation times varied downward from 146 to $53 \mathrm{msec}$ in eight regular steps.

\section{Method}

Participants. Fifteen participants took part in this experiment, including 12 females and 3 males; their ages ranged from 18 to 25 years (mean age $=20.6$ years). All the participants were members of Georgetown University; they were right-handed and had normal or corrected-to-normal vision; they had not taken part in the previous experiments. The participants were paid \$7 per hour for their participation.

Stimuli and Procedure. This experiment was run on a different experimental setup than were the first two experiments, resulting in minor modifications of the method, as noted below. The display was flashed on a Mitsubishi (Diamond Pro 21-TX) monitor. All the visual angles were kept identical to those used in Experiment 1, but the participants were now sitting 20 in. from the monitor.

The stimuli consisted of two perceptual groups composed of geometrical shapes. High-load displays consisted of hierarchical shapes similar to those used in Experiment 1. Low-load displays consisted of nonhierarchical shapes, whose size was matched to that used in the high-load displays. By analogy with the high-load condition, the small shapes in the low-load displays will be termed local, and the larger ones global (Figure 4). The targets were always local; the distractors were always located in the nontarget shape, at either the global or the local level. Presentation times varied from 146 to $53 \mathrm{msec}$ in $13.3-\mathrm{msec}$ steps and were presented randomly mixed. High- and low-load displays were administered in separate blocks, with the order of blocks randomized across participants. The high-load blocks included five experimental conditions (global/local $\times$ compatible/incompatible, plus one neutral condition) and the low-load block included six experimental conditions (global/local $\times$ compatible/incompatible/neutral). The neutral stimuli are shown in Appendix C.

The participants were trained at the beginning of each block by performing two sets of 12 trials. The first 3 trials were performed at a slower speed, so that the participants could get familiar with the experiment. The high-load blocks comprised 480 trials ( 96 trials per condition); the low-load blocks comprised 576 trials ( 96 trials per condition). Every 48 trials, the participants were given feedback about their mean accuracy and speed. The phrase "Press the space bar when ready" prompted the participants to press the space bar to initiate a trial. Following the initiation of a trial, a fixation point appeared in the center of the screen for $500 \mathrm{msec}$, immediately followed by the stimuli for one of the experimental display durations. The intertrial interval was set at $500 \mathrm{msec}$ after the execution of the response. The experiment lasted about $1 \mathrm{~h} 10 \mathrm{~min}$. The participants used the keyboard to make their responses. The participants were required to press the $n$ key with their index fingers for the target shape square and the $m$ key with their middle fingers for the target shape diamond. The procedure was otherwise similar to that in Experiment 1.

In addition, the participants were first tested on a block of lowload displays in which the distractor shape was always neutral and the target shape was either local or global. This test block contained 256 trials; the participants were given feedback about their mean accuracy and speed every 32 trials. The purpose of this test block was to establish that local shapes were harder than global ones in the low-load displays, since it is the case in the high-load displays. Analysis of RTs and percent error confirmed that local shapes were harder to process than global shapes $[M D=31 \mathrm{msec} ; F(1,14)=41.3$, $\left.M S_{\mathrm{e}}=315.7, p<.0001\right]$. This result will not be discussed further.

\section{Results}

As in Experiments 1 and 2, RTs outside two standard deviations were discarded from the analysis $(4.5 \%$ of the trials). The results are summarized in Table 3; mean RTs and percent error for neutral trials are given in Appendix D. In this experiment, as well as in Experiment 4, error analyses led to a pattern of results similar to that for RT analyses and will not be discussed further.

A $2 \times 2 \times 2 \times 8$ ANOVA was carried out, with perceptual load (high/low), distractor level (global/local), compatibility (compatible/incompatible), and presentation times ( $146 \mathrm{msec} / 133 \mathrm{msec} / 120 \mathrm{msec} / 106 \mathrm{msec} / 93 \mathrm{msec} /$ $80 \mathrm{msec} / 66 \mathrm{msec} / 53 \mathrm{msec}$ ) as factors. The mean RT was longer for high-load than for low-load displays [566 vs. $\left.514 \mathrm{msec} ; F(1,14)=28.8, M S_{\mathrm{e}}=22,184.8, p<.0001\right]$, confirming that the task was more difficult in high-load displays. The mean RT was longer for global than for local 
Table 3

Mean Reaction Times (RTs, in Milliseconds) and Error Rates (as Percentages) as a Function of Shape Complexity in Experiment 3

\begin{tabular}{|c|c|c|c|c|}
\hline \multirow{2}{*}{$\begin{array}{l}\text { Distractor Level } \\
\text { and Compatibility }\end{array}$} & \multicolumn{2}{|c|}{ Low Load } & \multicolumn{2}{|c|}{ High Load } \\
\hline & $\mathrm{RT}$ & Error & RT & Error \\
\hline \multicolumn{5}{|l|}{ Global } \\
\hline Compatible & 504 & 4.7 & 582 & 8.7 \\
\hline Incompatible & 533 & 6.6 & 563 & 4.4 \\
\hline Compatibility effect & $+29 t$ & $+1.9^{*}$ & $-19 \dagger$ & $-4.3 t$ \\
\hline \multicolumn{5}{|l|}{ Local } \\
\hline Compatible & 502 & 3.8 & 550 & 3.8 \\
\hline Incompatible & 519 & 5.6 & 569 & 5.9 \\
\hline Compatibility effect & $+17 \ddagger$ & +1.8 & $+19 t$ & $+2.1^{*}$ \\
\hline
\end{tabular}

${ }^{\circ} p<.05, \quad{ }^{\dagger} p<.01 . \quad{ }^{\ddagger} p<.001$.

distractors [545 vs. $535 \mathrm{msec} ; F(1,14)=18.1, M S_{\mathrm{e}}=$ $1,394.5, p<.0008$ ]; thus, as was previously observed, global distractors interfered with the target task more than did local distractors. The mean RT was faster for compatible distractors, revealing an overall PCE [534 vs. $\left.546 \mathrm{msec} ; F(1,14)=30.5, M S_{\mathrm{e}}=1,064.8, p<.0001\right]$. The effect of compatibility interacted, however, with perceptual load, as well as with distractor level $[F(1,14)=28.9$, $M S_{\mathrm{e}}=1,123.7, p<.0001$, and $F(1,14)=13.9, M S_{\mathrm{e}}=$ $704.3, p<.0022$, respectively]. The finding of different effects of compatibility as perceptual load was manipulated was consistent with the proposal that the size of the NCE should vary with display load. The triple interaction between compatibility, perceptual load, and distractor level further confirmed different effects of compatibility across the four distractor conditions $[F(1,14)=86.3$, $\left.M S_{\mathrm{c}}=434.5, p<.0001\right]$. This result led to separate ANOVAs for each of these conditions, with presentation time and compatibility as factors. The time of presentation did not interact with any of the effects studied ( $p s>$ $.07)$ and will not be discussed further. For high-load displays with global distractors, the mean RT was longer for compatible distractors, confirming the expected $\mathrm{NCE}$ $\left[M D=-19 \mathrm{msec} ; F(1,14)=15.1, M S_{\mathrm{e}}=1,421, p<\right.$ $.0017]$. For high-load displays with local distractors, the mean RT was faster for compatible distractors, revealing a PCE $\left[M D=+19 \mathrm{msec} ; F(1,14)=39.5, M S_{\mathrm{e}}=541.0\right.$, $p<.0001]$. This finding replicated previous reports in the literature (Paquet, 1992; Paquet \& Merikle, 1988); it was, however, unexpected, given the tendency for NCEs that was observed from similar between-group local distractors in Experiments 1 and 2. For low-load displays, the mean RT was faster for compatible distractors, whether at the global or the local level $[M D=+29 \mathrm{msec}, F(1,14)=$ 55.7, $M S_{\mathrm{e}}=930.0, p<.0001$, and $M D=+17 \mathrm{msec}$, $F(1,14)=40.6, M S_{\mathrm{e}}=435.5, p<.0001$, respectively]. This result replicated the standard facilitation by compatible distractors, as compared with incompatible ones, described in the literature on the flanker compatibility effect (Eriksen \& Eriksen, 1974).

Presentation time showed a main effect in the omnibus $2 \times 2 \times 2 \times 8$ ANOVA $\left[F(7,98)=2.8, M S_{\mathrm{e}}=879.2, p<\right.$
$.01]$. RTs tended to increase as the presentation time decreased. This effect is, however, hard to interpret, since the increase was not linear. Presentation time interacted with distractor level $\left[F(7,98)=2.1, M S_{\mathrm{e}}=526.2, p<\right.$ $.045]$, but not with compatibility or with perceptual load $(p s>.5)$. Interestingly, the triple interaction between presentation time, perceptual load, and compatibility was significant, as well as the quadruple interaction between all the factors $\left[F(7,98)=3.2, M S_{\mathrm{e}}=785.4, p<.0045\right.$, and $F(7,98)=2.2, M S_{\mathrm{e}}=616.3, p<.045$, respectively]. Although interactions between more than two factors are hard to interpret, these interactions reflected, in part, different patterns of compatibility effects in low-load and high-load displays as presentation time was varied. In particular, whereas compatibility effects were quite similar across presentation times in low-load displays, they showed more variation in high-load displays, especially when the distractor was global (Figure 5). This finding is in line with the report of Van Leeuwen and Bakker (1995) that the size of the NCE is highly sensitive to presentation times. Different presentation times were included, to ensure that the lack of an NCE in the low-load display was not due to the use of a specific presentation time. Accordingly, no trace of an NCE was observed in the low-load condition, even at the shortest presentation times.

Overall, these results support the proposal that perceptual load affects the size of the NCE. However, since a significant NCE was only observed from global distractors in this experiment, this proposal was best tested by considering global and local distractors separately. ANOVAs with perceptual load and compatibility as factors were carried out separately for the global and the local distractors. For the global distractors, a main effect of perceptual load indexed by longer RTs for high-load displays confirmed that target selection was harder when the shapes were complex hierarchical structures $[F(1,14)=$ $\left.28.3, M S_{\mathrm{e}}=1,496.2, p<.0001\right]$; more to the point, perceptual load and compatibility interacted, confirming different compatibility effects as perceptual load was varied $\left[F(1,14)=59.8, M S_{\mathrm{e}}=143.3, p<.0001\right]$. For the local distractors, the main effect of perceptual load was significant, confirming that the task was more difficult for high-load displays $\left[F(1,14)=25.9, M S_{\mathrm{e}}=1,424.8\right.$, $p<.0002]$. The main effect of compatibility was reliable, owing to an overall PCE $\left[F(1,14)=87.4, M S_{\mathrm{e}}=\right.$ $55.4, p<.0001]$. Load and compatibility did not interact, indicating similar PCEs across perceptual loads $(p>.8)$.

\section{Discussion}

The results of Experiment 3 confirm the hypothesis that the NCE decreases as the target task becomes easier and requires less selective attention. The significant interaction between perceptual load and compatibility for the global distractors supported this prediction. The global distractors led to a smaller NCE in low-load displays than in high-load displays. In fact, positive, rather than 


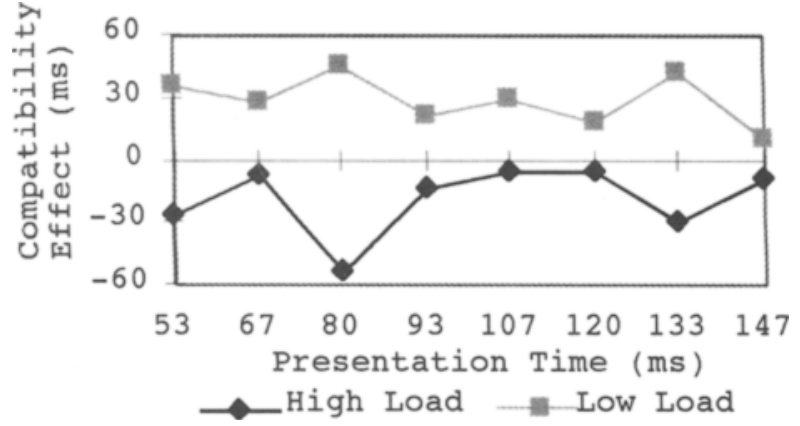

Figure 5. Compatibility effects from global distractors as a function of the presentation times in Experiment 3. Negative compatibility effects were observed for high-load displays, whereas positive compatibility effects were observed for low-load displays.

negative, compatibility effects were observed in lowload displays. The possibility that these PCEs were due to an inadequate choice of the presentation times appears highly unlikely. Eight different presentation times were used in Experiment 3; PCEs were observed for low-load displays for all the individual presentation times, even at the shortest ones (Figure 5). Thus, the pattern of compatibility effects in the low-load condition cannot be easily attributed to a different sensitivity to display duration. The robust PCEs observed in low-load displays are consistent with Lavie's (1995) proposal that the positive effects of compatibility should be strongest when the target task is easy and requires little selective attention. The claim that the target task is easier in low-load displays is supported by the finding of shorter RTs and fewer errors for low-load displays than for high-load displays.

Experiment 3 confirms the robustness of the NCE. The global distractors in high-load displays led to longer RTs when compatible. However, the presence of a PCE from the local distractors in high-load displays was unexpected, given the results of Experiments 1 and 2 for identical or similar configurations. It is possible that the striking symmetry in compatible local distractor displays was strong enough in Experiment 3 to overcome efficiently other conflicting parsing cues that indicated that the target and the distractors should be considered separately (such as the distinct gray backgrounds and the task requirements). The view that cues indicating separate groups were weaker in Experiment 3 is supported by the observation that the NCE from the global distractors was smaller than those in Experiments 1 and 2.

We have proposed that, under conditions in which the target task requires selective attention, the type of compatibility effect (negative, positive, or none) is influenced by the early parsing stages of the display. The NCE is attributed to a competition between conflicting parsing cues in the global compatible condition. Whereas shape similarity between targets and distractors tends to induce grouping, the different color and distinct gray backgrounds of the target and the distractor groups indicate that they should be segregated. The first three experiments documented the importance of a target task that requires selective attention in the manifestation of the NCE. In Experiment 4, the proposal that the NCE arises from a conflict between different segmentation cues when the target task is difficult was tested further. Experiment 4 replicated Experiment 3, except for an increase in luminance contrast. The efficiency of the early stages of segmentation, such as edge detection, is known to be influenced by luminance contrast (Nothdurft, 1994). The presence of high-contrast boundaries in Experiment 4 should allow the segmentation of the visual display to proceed faster and should facilitate target selection. The hypothesis that the NCE is due to a delay during the segmentation of compatible displays leads one to predict that it should be reduced with the high-luminance contrast of Experiment 4.

\section{EXPERIMENT 4}

Experiment 4 was identical to Experiment 3, except for a higher brightness and contrast of the displays. We predicted that the NCE observed with the complex, highload displays of Experiment 3 should be reduced when a high-luminance contrast was used. In contrast, the PCE observed with low-load displays in Experiment 3 was expected to display little sensitivity to this manipulation, since it was believed to occur mainly during response selection.

\section{Method}

Participants. Fifteen participants took part in this experiment, including 9 females and 6 males; their ages ranged from 18 to 22 years (mean age $=19.6$ years). All the participants were drawn from the same population as that in Experiment 3. None had participated in the previous experiments.

Stimuli and Procedure. The stimuli and procedures were identical to those in Experiment 3. The only difference was that the monitor settings were changed so that the brightness and contrast of the monitor were increased from $55 \%$ and $60 \%$, respectively, to $100 \%$ and $100 \%$. Luminance levels for the different components of a display are listed below in comparison with those of Experiment 3. The overall background was a homogeneous $82 \mathrm{~cd} / \mathrm{m}^{2}$ area (vs. $18 \mathrm{~cd} / \mathrm{m}^{2}$ in Experiment 3); the gray circle background had a luminance of $15 \mathrm{~cd} / \mathrm{m}^{2}$ (vs. $\left.1.9 \mathrm{~cd} / \mathrm{m}^{2}\right)$; the black shapes had a luminance

Table 4

Mean Reaction Times (RTs, in Milliseconds) and

Error Rates (as Percentages) as a Function of Perceptual Load in Experiment 4

\begin{tabular}{lccccc}
\hline \multirow{2}{*}{$\begin{array}{c}\text { Distractor Level } \\
\text { and Compatibility }\end{array}$} & \multicolumn{2}{c}{ Low Load } & & \multicolumn{2}{c}{ High Load } \\
& RT & Error & & RT & Error \\
\hline Global & & & & \\
$\quad$ Compatible & 469 & 6 & & 490 & 8.7 \\
$\quad$ Incompatible & 496 & 12.6 & & 507 & 8.8 \\
$\quad$ Compatibility effect & $+27 \ddagger$ & $+6.6 \dagger$ & $+17^{*}$ & +0.1 \\
Local & & & & \\
$\quad$ Compatible & 468 & 4.9 & & 482 & 5.2 \\
$\quad$ Incompatible & 482 & 8.6 & & 501 & 10.0 \\
Compatibility effect & $+14 \ddagger$ & $+3.7 \dagger$ & $+19 \ddagger$ & $+4.8^{*}$ \\
\hline${ }^{*} p<.05 . \quad{ }^{\dagger} p<.01 . \quad{ }^{\ddagger} p<.001$. & & &
\end{tabular}


of $4.8 \mathrm{~cd} / \mathrm{m}^{2}$ (vs. $\left..38 \mathrm{~cd} / \mathrm{m}^{2}\right)$; and the white shapes had a luminance of $88 \mathrm{~cd} / \mathrm{m}^{2}$ (vs. $26 \mathrm{~cd} / \mathrm{m}^{2}$ )

\section{Results}

RTs outside two standard deviations were discarded from the analysis (4.1\% of the trials). The results of Experiment 4 are given in Table 4; mean RT and percent error for neutral trials are given in Appendix D.

As for Experiment 3, a $2 \times 2 \times 2 \times 8$ ANOVA was carried out, with perceptual load (low/high), distractor level (global/local), compatibility (compatible/incompatible), and presentation times $(146 \mathrm{msec} / 133 \mathrm{msec} / 120 \mathrm{msec} /$ $106 \mathrm{msec} / 93 \mathrm{msec} / 80 \mathrm{msec} / 66 \mathrm{msec} / 53 \mathrm{msec}$ ) as factors. In contrast to what was observed in Experiment 3, no significant effect of presentation time was observed (all $p$ values in which presentation time was a factor were greater than .1). The mean RT was faster for compatible distractors, confirming an overall robust PCE [477 vs. $\left.497 \mathrm{msec} ; F(1,14)=55.7, M S_{\mathrm{e}}=1,626.5, p<.0001\right]$. No other significant effects were observed (all $p s>.15$ ), except for the triple interaction between perceptual load, distractor level, and compatibility, which led us to analyze the four distractor conditions separately $[F(1,14)=9.0$, $\left.M S_{\mathrm{e}}=434.8, p<.0094\right]$. In these separate ANOVAs, with presentation time and compatibility as factors, the effect of presentation time did not reach significance $(p s>.55)$ and will not be further discussed. For highload displays, the mean RT was faster for compatible distractors, whether at the global or the local level, revealing PCEs $\left[M D=+17 \mathrm{msec}, F(1,14)=8.5, M S_{\mathrm{e}}=2,096.7\right.$, $p<.012$, and $M D=+19 \mathrm{msec}, F(1,14)=23.56, M S_{\mathrm{e}}=$ $937.6, p<.0003$, respectively]. Thus, unlike in the three previous experiments, a reliable PCE was observed for the global distractors in the between-group, high-load display condition. For low-load displays, global and local distractors led to PCEs, as was previously observed $[M D=$ $+27 \mathrm{msec}, F(1,14)=41.3, M S_{\mathrm{e}}=1,120.4, p<.0001$, and $M D=+14 \mathrm{msec}, F(1,14)=22.9, M S_{\mathrm{e}}=1,120.4, p<$ .0003 , respectively].

The finding of a PCE, rather than an NCE, for the global distractors in the between-group, high-load displays condition supported the claim that the size of the NCE would decrease as target selection and display segmentation were facilitated by increased contrast and luminance in the display. This proposal could, however, be assessed directly by comparing the compatibility effects for highload global distractors as a function of the different monitor settings of Experiments 3 and 4 . A $2 \times 2$ ANOVA was carried out, with compatibility as a within-participant factor and monitor settings (Experiment 3/Experiment 4) as a between-subjects factor. The mean RT was faster for the monitor settings in Experiment 4 than for the monitor settings in Experiment 3, confirming the working hypothesis that higher contrast and luminance facilitated target selection $\left[499\right.$ vs. $572 \mathrm{msec} ; F(1,28)=8.6, M S_{\mathrm{e}}=$ $9,311.9, p<.0067]$. The main effect of compatibility was not significant $(p>.8)$, but a significant interaction between monitor settings and compatibility was observed, establishing significant changes in the NCE size between Experiments 3 and $4\left[F(1,28)=20.5, M S_{\mathrm{e}}=226.8\right.$, $p<.0001]$. This pattern of results confirmed the claim that the size of the NCE was influenced by early visual cues, such as display luminance and brightness.

Similar analyses were performed on the other distractor conditions to assess the effect of contrast/luminance changes on the other compatibility effects. For high load with local distractors, the mean RT was again faster for the monitor settings in Experiment 4 than for the monitor settings in Experiment 3, reenforcing the hypothesis that higher contrast and luminance facilitated target selection in the high-load displays [ 492 vs. $560 \mathrm{msec} ; F(1,28)=8.3$, $\left.M S_{\mathrm{e}}=8,388.7, p<.0076\right]$. The mean RT was faster for compatible distractors, indicating an overall PCE [516 vs. $\left.535 \mathrm{msec} ; F(1,28)=56.8, M S_{\mathrm{e}}=95.7, p<.0001\right]$. Monitor settings and compatibility did not interact $(p>8)$.

For low-load displays, the pattern of results was similar for the global and the local distractors. Only a main effect of compatibility was observed, owing to faster RTs in the compatible condition [global distractors: 486 vs. $515 \mathrm{msec}, F(1,28)=100.7, M S_{\mathrm{e}}=120.6, p<.0001$; local distractors: 485 vs. $500 \mathrm{msec}, F(1,28)=60.0$, $M S_{\mathrm{e}}=58.5, p<.0001 ;$ all other $\left.p \mathrm{~s}>.1\right]$. The lack of a monitor-setting effect suggested that changes in contrast did not significantly affect target selection. This result accords well with our proposal that target selection was already quite easy under the low-load conditions of Experiment 3 .

\section{Discussion}

As contrast and brightness were increased, the NCE observed in the high-load displays was significantly reduced. The finding that low-level visual information, such as brightness and contrast, can significantly influence the NCE supports the view that the NCE occurs during the early stages of visual processing. On the contrary, the PCE observed in the low-load displays remained unchanged as contrast and brightness were manipulated. This finding is consistent with the proposal that the PCE arises mainly at the stage of response selection. It is worth noting that the effect of monitor settings was significant for high perceptual load displays, but not for low-load displays. Thus, changes in the efficiency of the segmentation facilitated the target task only in high-load displays. This finding lends further support to the proposal that the target selection process is most influenced by early visual cues when the task is difficult. The results of Experiment 4 support the view that the NCE is under the control of early segmentation cues and strengthen the proposal that PCE and NCE occur at different stages of processing.

\section{GENERAL DISCUSSION}

Experiment 1 established an NCE, during which distractors hindered target processing more when they were compatible than when they were incompatible. We have proposed that, when the target task is difficult and requires 
selective attention, the type of compatibility effect (negative, positive, or none) will be under the influence of segmentation cues in the display. In this view, the NCE is observed when conflicting segmentation cues in the compatible condition delay the distribution of attention over the display. Experiments 2 and 3 confirmed the proposal that the NCE effect is observed when the target task is difficult. By extending the NCE to configurations with two embedded hierarchical levels, Experiment 2 established that the NCE generalized to other configurations beyond that observed to lead to an NCE in Experiment 1. Experiment 3 compared high-load displays in which the target task was difficult, with low-load displays, in which the target task was easy. The finding of a significant NCE for high-load displays but a PCE for low-load displays supported the claim that the NCE occurs when the target task is difficult and requires selective attention. Experiment 4 was identical to Experiment 3, except that target selection and display segmentation were facilitated by increasing the contrast and the brightness of the displays. High-load displays that led to an NCE in Experiment 3 exhibited positive effects of compatibility in Experiment 4. This result supported the proposal that the NCE is an early perceptual effect resulting from a delay in the allocation of attention in the compatible condition. This delay appears to be due to a competition between different grouping interpretations of the display. In contrast, the PCE observed for low-load displays in Experiment 3 was not affected by the brightness and contrast manipulation of Experiment 4, supporting the view that the PCE arises largely from response-related processes. These results lend additional support to the proposal that the NCE and the PCE occur at different stages of processing.

Our studies confirmed the robustness of the PCE in the standard global-to-local Navon configuration, in which targets and distractors are made up of each other. The use of configurations with two embedded hierarchical levels in Experiment 2 allowed us to test the global-to-local effect beyond the standard configuration. The lack of a compatibility effect from within-group global distractors in Experiment 2 contrasted with the robustness of the standard Navon effect. The size of the Navon effect might be dependent on the configural relationships between local and global levels. The colinearity and symmetry introduced in the compatible Navon configuration may be responsible for the robustness of the standard PCE.

The finding of a reliable NCE confirms and extends the reports of Briand (1994) and Van Leeuwen and Bakker (1995). We have proposed that the NCE arises because compatible distractors induce grouping by similarity while other cues in the display indicate that the target and the distractors should not be grouped. This view holds that the NCE should be strongest when the target and the distractors act as separate groups, rather than as parts of the same group. Accordingly, in our studies, as well as in those of Briand and of Van Leeuwen and Bakker, global distractors that did not belong to the same group as the target led to the strongest NCE, whereas little or no reli- able NCE was observed from within-group distractors. One may ask why the NCE has not been reported before, given the wealth of studies of the flanker effect. This series of experiments suggests a few possible explanations. Experiment 4 illustrated how the size of the NCE can be dramatically altered by the manipulation of low-level visual information, such as brightness and contrast. Little has been said about the status of these factors in related studies by Briand (1993), Paquet (1992; Paquet \& Appleby, 1988; Paquet \& Appleby, 1988; Paquet \& Merikle, 1988), and Van Leeuwen and Bakker. Furthermore, the report by Van Leeuwen and Bakker, that the size of the NCE was dependent on display duration, may also account for the variability in the manifestation of the NCE. Note that the rather small effects of display duration in Experiment 3 may be due to a number of procedural differences. In particular, in Van Leeuwen and Bakker's experiment, the target position was fixed throughout, allowing participants to know ahead of time where to focus attention. In contrast, in our experiments, the target location was not known ahead of time. The distribution of attentional resources when the target location is fixed may be less sensitive to segmentation cues and more sensitive to display duration.

The proposal that the type of compatibility effect (negative, positive, or none) is under the influence of early visual cues in the display is supported by other studies in the literature. For instance, the PCE has been shown to diminish in strength as target and distractors get harder to group together (Baylis \& Driver, 1992; Kramer \& Jacobson, 1991; Miller, 1991). The locus of the compatibility effect during the flanker paradigm has also been studied, using electrophysiological indices (Coles, Gratton, Bashore, Eriksen, \& Donchin, 1985; Fournier, Scheffers, Coles, Adamson, \& Abad, 1997). The use of the $\mathrm{P} 300$ and of the lateralized readiness potential allows for the evaluation of the respective contributions of early visual processes and response-related processes to the compatibility effect. These studies indicated that not only response-related processes, but also early visual processes contribute to the compatibility effect. In addition, the results of Fournier et al. suggested that the relative contribution of each process can be varied as one manipulates early visual cues, such as the feature similarity between the target and the distractors. In particular, these authors reported a greater contribution of early visual processes to the compatibility effect when the display contained different elements that had to be segregated and when it was difficult to do so because of their similarity. This result supports the proposal that emerged from our studies that the contribution of early visual processes to the compatibility effect is maximized when the task is difficult and requires selective attention. This view accords well with Lavie's theory of visual selection, ${ }^{1}$ which holds that when target selection exhausts the attentional resources, the target becomes selectively attended and the PCE diminishes (Lavie, 1995; Lavie \& Cox, 1997). Under these conditions, the contribution of responserelated processes to the compatibility effect is reduced, 
allowing early visual effects, such as the NCE, to be revealed.

\section{Relationship to Other Phenomena}

The NCE bears a strong resemblance to the repeatedletter inferiority effect (Bjork \& Murray, 1977; Egeth \& Santee, 1981; Santee \& Egeth, 1980). In this paradigm, a stimulus matrix containing two letters, with all the remaining positions filled with a dummy character is briefly presented. At the end of the trial, one of the columns in the display is cued and participants have to decide which of the two target letters were presented in the cued column. Participants' performance is less accurate when the other distractor letter in the display is identical to the cued letter than when it is different. The effect is still observed for visually different distractors, if they share the same name as the target (e.g., Aa; Egeth, \& Santee, 1981). Thus, the repeated-letter inferiority effect, like the NCE, is observed with displays of brief duration and relatively high perceptual load and from distractors that are not visually identical but share the same name as the target. Although the repeated-letter inferiority effect has been proposed to be due to interference at a relatively early stage of perceptual processing, it is still unclear why the repeated-letter inferiority effect should occur.

The NCE also bears a strong resemblance with the report by Van Leeuwen and Bakker (1995) of a negative interference effect. These authors proposed that negative and positive interference effects should be understood as being the by-product of attention gradually expanding over the display. Initially, attention is focused on the target, allowing information at the target location to be integrated and processed. During that time, other irrelevant information in the display is suppressed, leading to a decrement in performance for compatible distractors. Once the figure on which attention was allocated has been integrated, attention is stretched beyond that figure to integrate more components of the stimulus. As distractors get integrated with the target, PCEs are observed. This account fits well with our report that NCEs are observed when selective attention is needed, whereas PCEs are observed otherwise. It will be important for future research to understand why suppression is needed early on, whereas facilitation occurs later on.

\section{Conclusion}

The NCE seems best explained as a general property of target/distractor interaction when attentional resources have been exhausted and selective attention is needed for target selection. Under these conditions, the efficiency of target selection is determined by the time it takes to selectively allocate attention to the target location. This process is believed to be under the control of cues as varied as luminance and orientation contrast, gestalt properties, and task requirements (Kramer et al., 1995; Vecera \& Farah, 1994). In contrast, the PCE seems best explained as a general property of the target/distractor interaction when attention can be allocated to both targets and distractors (Lavie \& Cox, 1997). Targets and distractors, when attended, are hypothesized to gain access to higher levels of processing, eventually leading to a competition during response selection between their different representations (Eriksen \& Eriksen, 1974; Kornblum \& Lee, 1995; Miller, 1982).

\section{REFERENCES}

Baylis, G. C.. \& Driver, J. (1992). Visual parsing and response competition: The effect of grouping factors. Perception \& Psychophysics, 51, 145-162.

BJork, E. L., \& MurraY, J. T. (1977). On the nature of input channels in visual processing. Psychological Review, 84, 472-484.

BRIAND, K. A. (1993). Efficient filtering of irrelevant global and local information when target level and location are random. Psychological Research, 55, 264-269.

BrIAND, K. A. (1994). Selective attention to global and local structure of objects: Alternative measures of nontarget processing. Perception \& Psychophysics, 55, 562-574.

Coles, M. G. H., Gratton, G., Bashore, T. R., Eriksen, C. W., \& DONCHIN, E. (1985). A psychophysiological investigation of the continuous flow model of human information processing. Journal of Experimental Psychology: Human Perception \& Performance, 11, 529 553.

EGETH, H. E., \& SANTEE, J. L. (1981). Conceptual and perceptual components of interletter inhibition. Journal of Experimental Psychology: Human Perception \& Performance, 7, 506-517.

ERIKSEN, B. A., \& ERIKSEN, C. W. (1974). Effects of noise letters upon the identification of a target letter in a nonsearch task. Perception \& Psychophysics, 16, 143-149.

Fagot, J., \& Deruelle, C. (1997). Processing of global and local visual information and hemispheric specialization in humans (Homo sapiens) and baboons (Papio papio). Journal of Experimental Psychology: Human Perception \& Performance, 23, 429-442.

Fournier, L. R., Scheffers, M. K., Coles. M. G. H., Adamson, A., \& ABAD, E. V. (1997). The dimensionality of the flanker compatibility effect: A psychophysiological analysis. Psychological Research, 60, 144-155.

Greaney, J., \& MaCRaE, A. W. (1992). The order of visual processing: Top-down, bottom-up, middle-out, or none of these? Bulletin of the Psychonomic Society, 30, 255-257.

KorNblum, S., \& LEe, J.-W. (1995). Stimulus-response compatibility with relevant and irrelevant stimulus dimensions that do and do not overlap with the response. Journal of Experimental Psychology: Human Perception \& Performance, 21, 855-875.

Kramer, A. F., Coles, M. G. H., \& Logan, G. D. (1995). Converging operations in the study of visual selective attention. Washington, DC: American Psychological Association.

Kramer, A. F., \& JaCOBSON. A. (1991). Perceptual organization and focused attention: The role of objects and proximity in visual processing. Perception \& Psychophysics, 50, 267-284.

Lavie, N. (1995). Perceptual load as a necessary condition for selective attention. Journal of Experimental Psychology: Human Perception \& Performance, 21, 451-468.

Lavie. N., \& Cox, S. (1997). On the efficiency of visual selective attention: Efficient visual search leads to inefficient distractor rejection. Psychological Science, 8, 395-398.

Miller, J. (1982). Divided attention: Evidence for co-activation with redundant signals. Cognitive Psvchology, 14, 247-279.

MILLER, J. ( 1991 ). The flanker compatibility effect as a function of visual angle, attentional focus, visual transients, and perceptual load: A search for boundary conditions. Perception \& Psychophysics, 49, 270-288

NAvon, D. (1977). Forest before the tree: The precedence of global feature in visual perception. Cognitive Psycholog, , 9, 353-383.

Navon, D. (1983). How many trees does it take to make a forest? Perception, 12, 239-254. 
NothDURFT, H.-C. (1994). Common properties of visual segmentation. In G. R. Bock \& J. A. Goode (Eds.), Ciba foundation symposium: Higher-order processing in the visual system (Vol. 184, pp. 245-268). Chichester, U.K.: Wiley.

PAQUET, L. (1992). Global and local processing in nonattended objects: A failure to induce local processing dominance. Journal of Experimental Psychology: Human Perception \& Performance, 18, 512-529.

PaqueT, L., \& Appleby, K. (1988). L'attention selective: L'effet du critère de selection. [Selective attention: Effect of selection criteria]. L'Annee Psychologique, 88, 179-195.

Paquet, L., \& Merikle, P. M. (1988). Global precedence in attended and nonattended objects. Journal of Experimental Psychology: Human Perception \& Performance, 14, 89-100.

SANTEE, J. L., \& EGeth, H. E. (1980). Interference in letter identification: A test of feature-specific inhibition. Perception \& Psychophysics, 27, 321-330.

VAN LeEUWEn, C., \& BaKKer, L. (1995). Stroop can occur without Garner interference: Strategic and mandatory influences in multidimensional stimuli. Perception \& Psychophysics, 57, 379-392.

Vecera, S. P., \& Farah, M. J. (1994). Does visual attention select objects or locations? Journal of Experimental Psychology, 123, 146-160.

YANTIS, S. (1995). Attentional capture in vision. In A. Kramer, M. Coles, \& G. Logan (Eds.), Converging operations in the study of visual selective attention (pp. 45-76). Washington, DC: American Psychological Association.

\section{NOTE}

1. Note that Lavie's experiments focus on the comparison of incompatible and neutral trials (Lavie, 1995; Lavie \& Cox, 1997). This measure does not take into account compatible trials and, so, cannot capture the NCE discussed in this paper. Our data, reanalyzed with the incompatible to neutral measure used by Lavie, are, however, consistent with her findings. As was expected, the incompatible to neutral difference was greatest when robust PCEs were observed and smallest when NCEs were observed.

\section{APPENDIX A}

Mean Reaction Times (RTs, in Milliseconds) and Error Rates (as Percentages) for Neutral Distractors in Experiments 1 and 2

\begin{tabular}{cccccc} 
& \multicolumn{2}{c}{ Experiment 1 } & & \multicolumn{2}{c}{ Experiment 2 } \\
\cline { 2 - 3 } \cline { 5 - 6 } & RT & Error & & RT & Error \\
\hline Neutral & 759 & 5 & & 721 & 4 \\
\hline
\end{tabular}

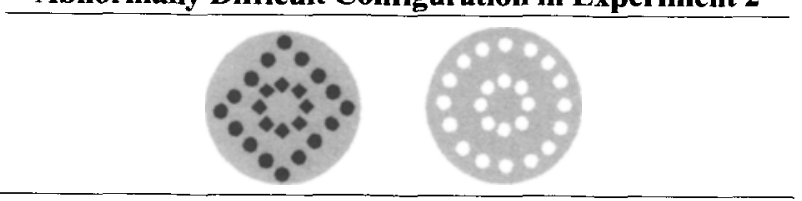

APPENDIX C

Examples of Neutral Stimuli for the Low-Load and High-Load Displays in Experiment 3

Low Load

Neutral Global

Neutral Local
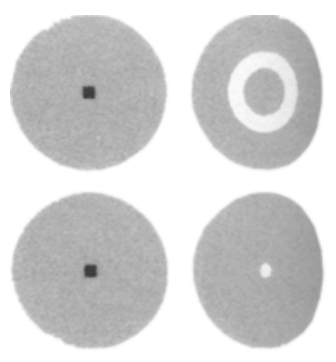

Neutra 1

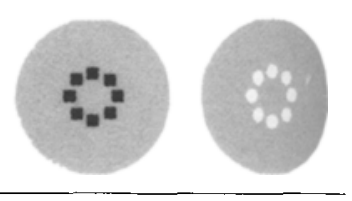

Mean Reaction Times (RTs, in Milliseconds) and Error Rates (as Percentages) for Neutral Distractors in Experiments 3 and 4

\begin{tabular}{|c|c|c|c|c|}
\hline & \multicolumn{2}{|c|}{ Experiment 3} & \multicolumn{2}{|c|}{ Experiment 4} \\
\hline & $\mathrm{RT}$ & Error & RT & Error \\
\hline \multicolumn{5}{|c|}{ Low Load } \\
\hline Neutral global & 501 & 4 & 465 & 5 \\
\hline Neutral local & 507 & 4 & 474 & 6 \\
\hline \multicolumn{5}{|c|}{ High Load } \\
\hline Neutral & 549 & 3.5 & 483 & 7 \\
\hline
\end{tabular}

(Manuscript received February 13, 1998; revision accepted for publication October 7,1998 .) 